\title{
Blau syndrome-related CARD15/NOD2 mutations are not linked to idiopathic uveitis in Spanish patients
}

\author{
Noelia Rodríguez-Pérez ${ }^{\mathrm{a}}$, Ana Aguinaga-Barrilero ${ }^{\mathrm{a}}$, Marina B. Gorroño-Echebarría ${ }^{\mathrm{b}}$, \\ Mercedes Pérez-Blas ${ }^{\mathrm{a}}$ and José M. Martín-Villa ${ }^{\mathrm{a}, *}$ \\ ${ }^{a}$ Inmunología, Facultad de Medicina, Universidad Complutense de Madrid, Madrid, Spain \\ ${ }^{\mathrm{b}}$ Servicio de Oftalmología, Hospital Universitario "Príncipe de Asturias", Alcalá de Henares, Madrid, Spain
}

\begin{abstract}
Uveitis is a clinical feature of the Blau syndrome, a disease linked to CARD15 (also referred to as NOD2) mutations. Three main mutations in this gene (R334W, R334Q and L469F) have been reported as Blau syndrome risk factors, a disease that manifests uveitis as one of its clinical features. However, little is known on the involvement of this gene in idiopathic uveitis. We thus sought to determine the frequency of these Blau-related CARD15 mutations in a cohort of Spanish patients with idiopathic uveitis. To this aim, 110 patients with idiopathic uveitis, followed at the Department of Ophtalmology of a tertiary hospital (Hospital Universitario Alcalá de Henares, Madrid. Spain) were enrolled. As a control population, 104 healthy subjects were used. DNA was extracted from blood samples and the Blau-related CARD15 mutations were analysed either by PCR-RFLP or direct DNA sequencing. None of the mutations studied was found in any of the individuals tested, whether diseased or healthy. It seems thus that Blau syndrome-related CARD15 mutations are not involved in idiopathic uveitis, a finding which allows us to suggest that the genetic aetiology of the idiopathic uveitis or the Blau-associated uveitis is different.
\end{abstract}

Keywords: Blau syndrome, CARD15, idiopathic uveitis, NOD2

\section{Introduction}

Idiopathic uveitis (uveitides) includes a group of inflammatory conditions which affect mainly the uveal tract of the eye, located between the sclera, conjunctiva and the anterior chamber on the outside, and the retina on the inside [13]. Uveitis can be further classified depending on the anatomic part of the eye affected in anterior, intermediate or posterior uveitis, and panuveitis (affecting the anterior chamber, vitreous and retina or choroid). The etiology of this condition is not well understood and both, genetic and environmental factors seem to be key factors in the disease appearance and

* Corresponding author: Prof. José M. Martín-Villa, Inmunología, Facultad de Medicina, Pabellón V, planta $4^{\mathrm{a}}$, Universidad Complutense de Madrid, 28040 Madrid, Spain. Tel.: +34 913941642; Fax: +34 913941641; E-mail: autoinmunidad@med.ucm.es. progression. Genes involved include the Major Histocompatibility Complex of humans (HLA-B27), cytokine gene polymorphisms and chemokine receptor genes [10].

The caspase recruitment domain (CARD) 15 gene (also referred to as NOD2 gene) has been pointed as a risk susceptibility factor to develop Blau syndrome (MIM\#186580), a chronic inflammatory disorder characterized by skin rash, chronic symmetric arthritis, camptodactyly and recurrent uveitis [1].

The CARD15 gene is located on chromosome 16q12 and encodes a cytosolic protein termed nucleotide oligomerization domain $2(\operatorname{Nod} 2)$, involved in intracellular detection of bacterial components (muramyldipeptide, MDP). It is expressed mainly on monocytes, granulocytes and dendritic cells, and consists of several functional domains: two amino-terminal CARD domains, a central nucleotide binding domain 
(NBD) and 10 carboxi-terminal leucine rich repeat (LRR) domains. The LRR domains are involved in the interaction with the infecting pathogen [2], whereas the CARD domains enable the protein to induce apoptosis and the NF- $\kappa \mathrm{B}$ signalling pathway.

Three major CARD15 variants have been shown to predispose to Blau Syndrome [12], affecting residues 334 and 469 of the molecule and thus increasing basal $\mathrm{NF}-\kappa \mathrm{B}$ activity. These are a $1002 \mathrm{C} \rightarrow \mathrm{T}$ transition, responsible for the R334W mutation, where an arginine is replaced by a tryptophan, a $1003 \mathrm{G} \rightarrow$ A transition that causes the R334Q change where an arginine is replaced by a glutamine and a $1409 \mathrm{C} \rightarrow \mathrm{T}$ transition causing the $\mathrm{L} 469 \mathrm{~F}$ mutation where a leucine is substituted by a phenylalanine. All three mutations lay at exon 4 located in the NBD region of Nod2 and result in an increase in the activity of NF- $\kappa \mathrm{B}$.

Given the presence of uveitis in the Blau syndrome and the involvement of CARD15 gene mutations in the syndrome predisposition, we sought to investigate the three aforementioned mutations in a group of Spanish patients with idiopathic uveitis.

\section{Patients and methods}

\subsection{Patients}

One hundred and ten unrelated Spanish patients with idiopathic uveitis diagnosed according to standardized criteria [6] were included in the present study, and disclosed as follows: 70 were anterior uveitis (AU) patients, 18 intermediate uveitis (IU) and 22 posterior uveitis (PU). A more detailed description of this cohort of patients is given in a previous publication from our group [14]. Written consent was obtained from the all patients involved in this study. This research was approved by the Ethic Committee of the Institutions involved (Universidad Complutense de Madrid, Madrid and Hospital Universitario Príncipe de Asturias, Alcalá de Henares) and conforms with the Declaration of Helsinki. As a control group, DNA samples from 104 healthy race-matched volunteers (kindly provide by Dr. J.L. Vicario (Centro de Transfusión de la C.A.M, Madrid, Spain), were used.

\subsection{DNA extraction}

Blood samples were obtained by venopunction in EDTA-containing tubes. DNA was extracted by means of the DNAzol technique, according to the manufacturer's protocol. (DNAzol reagent, Carlsbad, CA, USA)

ANALYISIS OF R334W, R334Q AND L469F MUTATIONS (see Table 1).

\subsection{1. $R 334 W$ and $R 334 Q$ mutations}

Given the large number of samples to be studied, a quick analytical method was devised and, thus, these mutations were analysed by PCR-RFLP technique. DNA samples were PCR amplified using specific primers (forward 5' TGACGATGCGGACACTGTGC 3', and reverse 5' AACAGGACACGGTCAGGGTG $3^{\prime}$ ) and conditions (initial denaturation $95^{\circ} \mathrm{C} 10 \mathrm{~min}$., amplification 33 cycles: $94^{\circ} \mathrm{C} 30 \mathrm{sec} ., 57^{\circ} \mathrm{C} 30 \mathrm{sec}$., $72^{\circ} \mathrm{C} 30 \mathrm{sec}$., and a final extension step $72^{\circ} \mathrm{C} 10 \mathrm{~min}$.). Amplified products were subjected to DNA digestion with the MspI restriction enzyme $\left(37^{\circ} \mathrm{C}, 8\right.$ hours $)$ and the fragments obtained resolved using a $10 \%$ polyacrylamide gel. Bands were visualized using a silver staining procedure. Direct DNA sequencing was also used to assess this polymorphism, either as an alternative methodology (in 35 patients and 34 control subjects) or as a means to confirm results obtained by PCR-RFLP (in 8 patients and 6 control individuals). Sequencing reactions were carried out at the core DNA sequencing facility (C.I.B., Madrid, Spain) using the primers above mentioned

\subsubsection{L469F polymorphism}

A TaqMan SNP Genotyping Assay was designed to analyse this polymorphism (Applied Biosystems, Foster City, CA, USA) and run according to manufacturer's protocols, using an Applied Biosystems Real-Time PCR 7900 HT Fast System.

\subsection{Allele frequency}

Allele frequency of the mutations considered was calculated by direct gene counting.

\subsection{Statistical analysis}

The frequency of the different mutations in the group of patients was compared to that of the group of healthy subjects, using the Chi-square with Yates' correction test, or Fisher exact probability test when needed. A p value less than 0.05 was considered significant.

\section{Results}

All patients and control subjects analyzed for the mutations considered were wild type and none of the mutations described for Blau syndrome were found in any of the samples tested (Table 1). 
Table 1

CARD15 mutations studied analyzed, localization, method of detection and their frequencies in the groups studied

\begin{tabular}{ccccccc}
\hline Exon & Domain & SNP & $\begin{array}{c}\text { Aminoacid } \\
\text { change }\end{array}$ & $\begin{array}{c}\text { Method } \\
\text { analysis }\end{array}$ & $\begin{array}{c}\text { Patients }^{\text {a }} \\
(110)\end{array}$ & $\begin{array}{c}\text { Controls }^{(104)} \\
(104)\end{array}$ \\
\hline 4 & NBD & $1002 \mathrm{C} \rightarrow \mathrm{T}$ & R334W & PCR-RFLP, DNA Sequencing & $0 \%$ & $0 \%$ \\
4 & NBD & $1003 \mathrm{G} \rightarrow \mathrm{A}$ & R334Q & PCR-RFLP, DNA Sequencing & $0 \%$ & $0 \%$ \\
4 & NBD & $1409 \mathrm{C} \rightarrow \mathrm{T}$ & L469F & TaqMan SNP & $0 \%$ & $0 \%$ \\
\hline
\end{tabular}

${ }^{\text {a }}$ Patients disclosed as follows: 70 anterior uveitis; 18 intermediate uveitis; 22 posterior uveitis.

TOP

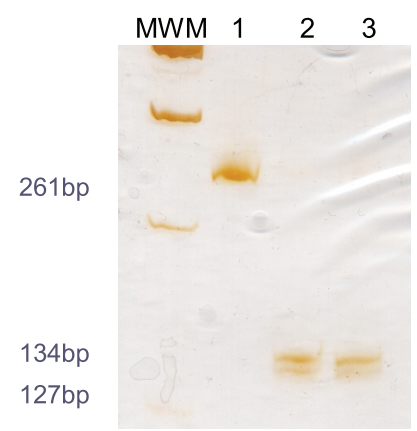

BOTTOM

TCCCATTCAGCTGCOGGCAGCTGCAGTGC
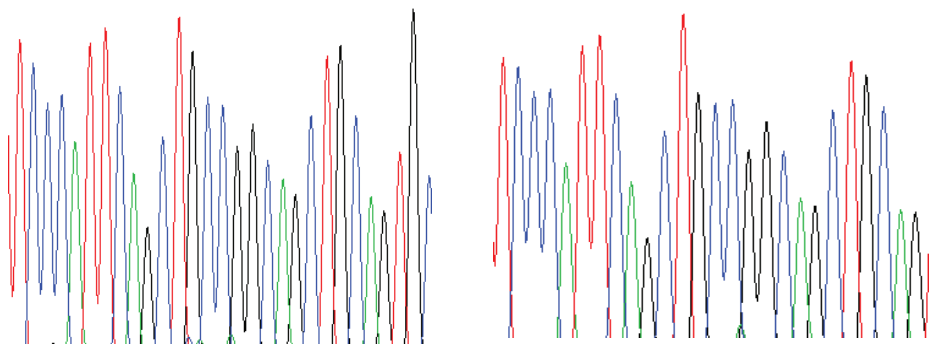

Fig. 1. TOP. Representative polyacrylamide gel (10\%) showing the PCR-RFLP result using MspI restriction enzyme. None of the samples analyzed showed the R334W or R334Q mutations (no enzyme cut). Lane 1 MWM, molecular weight marker. Lane 1, uncut amplification product (no restriction enzyme added) from a patient, showing a $261 \mathrm{bp}$ band. Lanes 2 and 3, amplification product digested with MspI from two different patients digested with MspI showing two bands of 134bp and 127bp, respectively, indicating a wild type allele (no base change). Samples in lanes 1 and 2 are from the same individual. BOTTOM. Chromatogram showing the DNA sequence of this region using the same individuals as above, confirming the results obtained by PCR-RFLP.

Figure 1A shows the PCR-RFLP pattern obtained when analyzing the 334 position. MspI digestion of amplified products yielded two bands of $134 \mathrm{bp}$ and $127 \mathrm{bp}$, indicating that no base changes were present in the DNA samples. The presence of a genetic variant would have rendered a single $261 \mathrm{bp}$ band. This result was further confirmed by DNA sequencing. The sequence obtained (Fig. 1B) was equal in all samples, whether from patients or control healthy individuals, and equal to the wild type sequence (GeneBank acces- sion number AJ303140.1HSA303140).

Thus, our results indicate that mutations involved in Blau syndrome are not risk factors for idiopathic uveitis.

\section{Discussion}

This is the first report carried out on the frequency of the Blau syndrome-associated CARD15 mutations 
in a large cohort of Spanish subjects, either diseased (idiopathic uveitis) or healthy. Thus, our data results may help fill data on the population frequency of these mutations, and may be useful in future research works.

The results herein presented show that the mutations involved in Blau syndrome are not associated to idiopathic uveitis. This suggests that the genetic etiology of the Blau-associated uveitis is different to that of idiopathic uveitis. Probably other genes may be more relevant in this latter type of uveitis, and, in fact, several reports in the literature have claimed the involvement of MHC (HLA-B27) or cytokine genes [10].

Our results match previous published data [11] that showed a lack of association between CARD15 mutations and uveitis status in a group of patients with sarcoidosis. Recently published results in mice [15] showed that CARD15 is the gene involved in granulomatous uveitis susceptibility. This finding does not contradict our results since, conceivably, the animal model and our patients would behave in an opposite manner with regard to CARD15-mediated uveitis: the $\mathrm{KO}$ mice are homozygous for a (recessive) loss-offunction mutation whereas the hypothetical uveitis patients sought in this study (and the Blau syndrome patients) are heterozygous for dominantly-acting, and probably gain-of-function mutations.

Different inflammatory conditions have been associated to mutations in the CARD15 gene. On the one hand Crohn's disease is linked to changes affecting mainly the LRR region of the molecule [9], altering the ability of the molecule to detect the MDP component of pathogens in blood, leading to an increased NF- $\kappa \mathrm{B}$ activation. On the other hand, the three most frequent mutations conferring susceptibility to Blau syndrome (analyzed in this work) are located in the NBD domain of the molecule, a different region of the protein. These mutations do not affect the sensor recognition capability of cells but, nevertheless, lead also to an increased $\mathrm{NF}-\kappa \mathrm{B}$ activation.

Interestingly, all the inflammatory conditions that show a tight linkage with CARD15 mutations (Blau syndrome, Crohn's disease, and early onset sarcoidosis) [7] show granuloma formation as a prominent clinical feature. However, other inflammatory conditions that show no or weak CARD15 association, such as ulcerative colitis [9], rheumatoid arthritis, ankylosing spondylitis, type I diabetes or systemic lupus erythematosus [5] lack this clinical feature. We further report here on another inflammatory condition (idiopathic uveitis) that, in most instances (and definitely in none of our patients), is not associated to granuloma forma- tion $[4,8]$ and that also fails to show any association with this gene, whether we analyze the NBD domain (as in the present work) or the LRR region of the molecule (as in a previous work from our group [14]). Some other pathologies, such as malignancies [5] or graft versus host $(\mathrm{GvH})$ disease in stem cell transplantation [3] have been effectively linked to CARD15 mutations and yet none of them is granulomatous. However, they are not autoinflammatory conditions and thus CARD15 may not be playing a role in granuloma formation in these settings.

It is then conceivable that the CARD15 mutations so far described in these regions may be involved in granuloma formation rather than in inflammation susceptibility. The tissue distribution of the CARD15 protein (macrophages), further supports this notion, since these cells are crucial in the granuloma development: continuous activation of the macrophage and increased $\mathrm{NF}-\kappa \mathrm{B}$ activity could lead to granulomas.

To unveil the role of CARD15 in uveitis susceptibility, it is required to sequence the whole gene in our cohort of patients and control subjects, a currently ongoing task in our laboratory.

\section{Acknowledgments}

This work was supported by a grant from La Fundación de Investigación Médica Mutua Madrileña Automovilista. N R-P and A. A-B are grant recipients from Universidad Complutense de Madrid. We are most grateful to Dr. E. Gómez de la Concha and his group (Servicio de Inmunolog ía, Hospital Clínico. Madrid) for help with the Real-Time PCR 7900 HT Fast System, and to Geno, Paloma, Pepi and Pilar (Oftalmología, Hospital Príncipe de Asturias) for their help in collecting blood samples.

\section{References}

[1] M.L. Becker and C.D. Rose, Blau syndrome and related genetic disorders causing childhood arthritis, Curr Rheumatol Rep 7 (2005), 427-433.

[2] L.L. Bourhis and C. Werts, Role of Nods in bacterial infection, Microbes Infect 9 (2007), 629-636.

[3] M. Granell, A. Urbano-Ispizua, J.I. Arostegui, F. FernandezAviles, C. Martinez, M. Rovira, J. Rius, S. Plaza, A. Gaya, A. Navarro, C. Talarn, E. Carreras, M. Monzo, E. Montserrat and J. Yague, Effect of NOD2/CARD15 variants in T-cell depleted allogeneic stem cell transplantation, Haematologica 91 (2006), 1372-1376.

[4] I.H. Hamade, H.N. Al Shamsi, H. Al Dhibi, C.B. Chacra, A.M. Abu El-Asrar and K.F. Tabbara, Uveitis survey in children, $\mathrm{Br}$ J Ophthalmol 93 (2009), 569-572. 
[5] L. Henckaerts and S. Vermeire, NOD2/CARD15 disease associations other than Crohn's disease, Inflamm Bowel Dis 13 (2007), 235-241.

[6] D.A. Jabs, R.B. Nussenblatt and J.T. Rosenbaum, Standardization of Uveitis Nomenclature (SUN) Working Group, Standardization of uveitis nomenclature for reporting clinical data. Results of the First International Workshop, Am J Ophthalmol 140 (2005), 509-516.

[7] N. Kambe, R. Nishikomori and N. Kanazawa, The cytosolic pattern-recognition receptor Nod2 and inflammatory granulomatous disorders, J Dermatol Sci 39 (2005), 71-80.

[8] J.D. Keenan, H.H. Tessler and D.A. Goldstein, Granulomatous inflammation in juvenile idiopathic arthritis-associated uveitis, J AAPOS 12 (2008), 546-550.

[9] S. Lesage, H. Zouali, J.P. Cezard, J.F. Colombel, J. Belaiche, S. Almer, C. Tysk, C. O’Morain, M. Gassull, V. Binder, Y. Finkel, R. Modigliani, C. Gower-Rousseau, J. Macry, F. Merlin, M. Chamaillard, A.S. Jannot, G. Thomas and J.P. Hugot, EPWG-IBD Group, EPIMAD Group, GETAID Group, CARD15/NOD2 mutational analysis and genotype-phenotype correlation in 612 patients with inflammatory bowel disease, Am J Hum Genet 70 (2002), 845-857.
[10] T.M. Martin and J.T. Rosenbaum, Genetics in uveitis, Int Ophthalmol Clin 45 (2005), 15-30.

[11] T.M. Martin, T.M. Doyle, J.R. Smith, D. Dinulescu, K. Rust and J.T. Rosenbaum, Uveitis in patients with sarcoidosis is not associated with mutations in NOD2 (CARD15), Am J Ophthalmol 136 (2003), 933-935.

[12] C. Miceli-Richard, S. Lesage, M. Rybojad, A.M. Prieur, S. Manouvrier-Hanu, R. Hafner, M. Chamaillard, H. Zouali, G. Thomas and J.P. Hugot, CARD15 mutations in Blau syndrome, Nat Genet 29 (2001), 19-20.

[13] S. Munoz-Fernandez and E. Martin-Mola, Uveitis, Best Pract Res Clin Rheumatol 20 (2006), 487-505.

[14] N. Rodriguez-Perez, A. Aguinaga-Barrilero, M.B. GorronoEchebarria, M. Perez-Blas and J.M. Martin-Villa, Analysis of Crohn's disease-related CARD15 polymorphisms in Spanish patients with idiopathic uveitis, Dis Markers 24 (2008), 111117.

[15] H.L. Rosenzweig, T.M. Martin, M.M. Jann, S.R. Planck, M.P. Davey, K. Kobayashi, R.A. Flavell and J.T. Rosenbaum, NOD2, the gene responsible for familial granulomatous uveitis, in a mouse model of uveitis, Invest Ophthalmol Vis Sci 49 (2008), 1518-1524. 


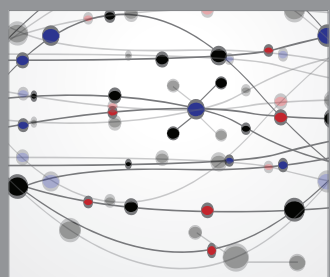

The Scientific World Journal
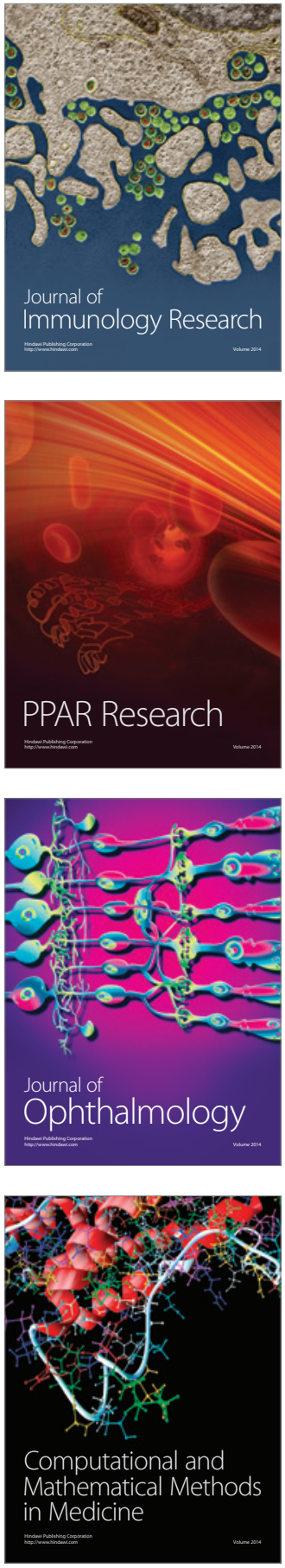

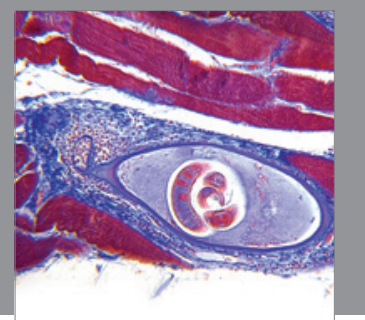

Gastroenterology

Research and Practice
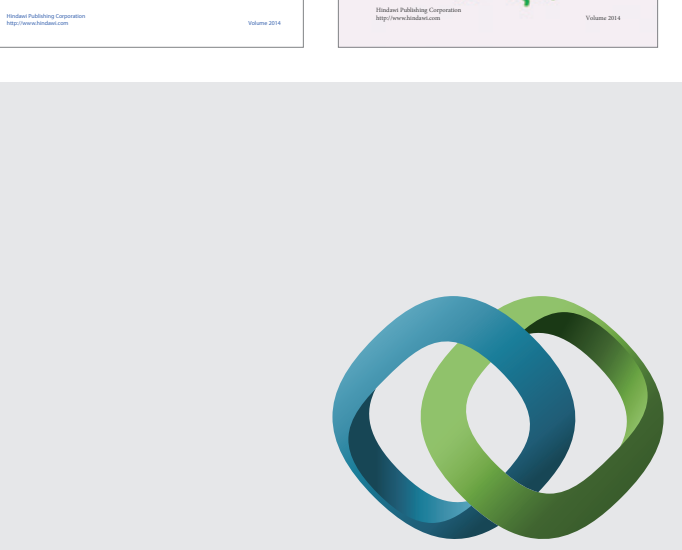

\section{Hindawi}

Submit your manuscripts at

http://www.hindawi.com
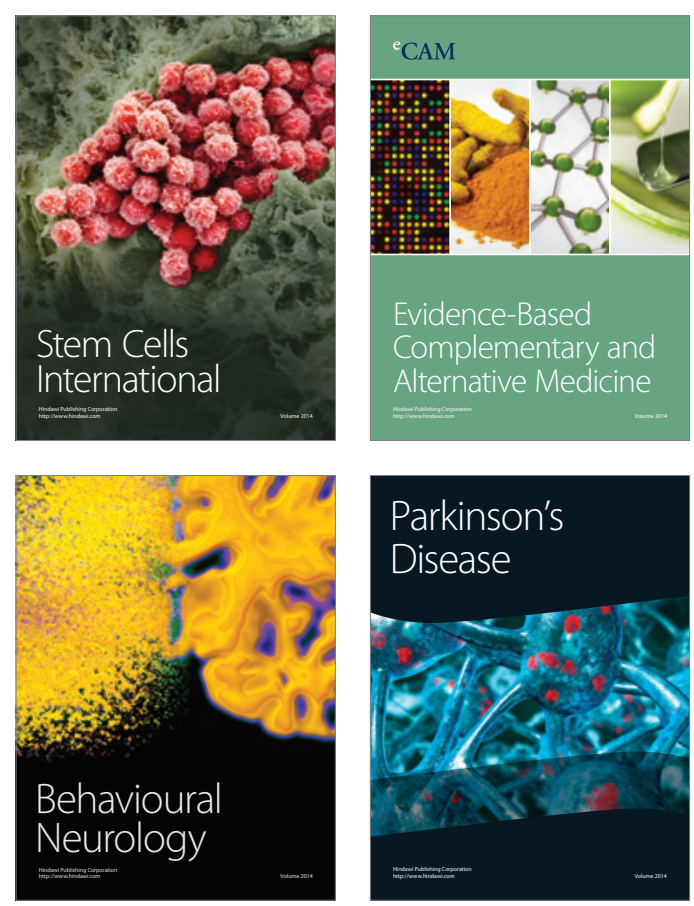

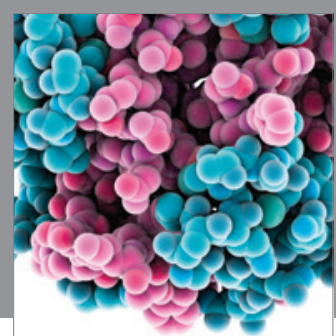

Journal of
Diabetes Research

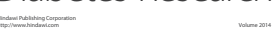

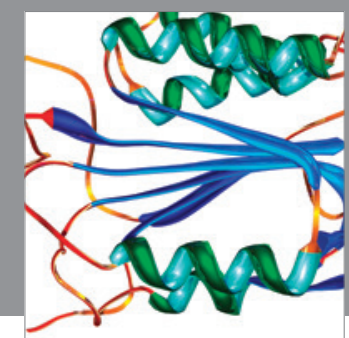

Disease Markers
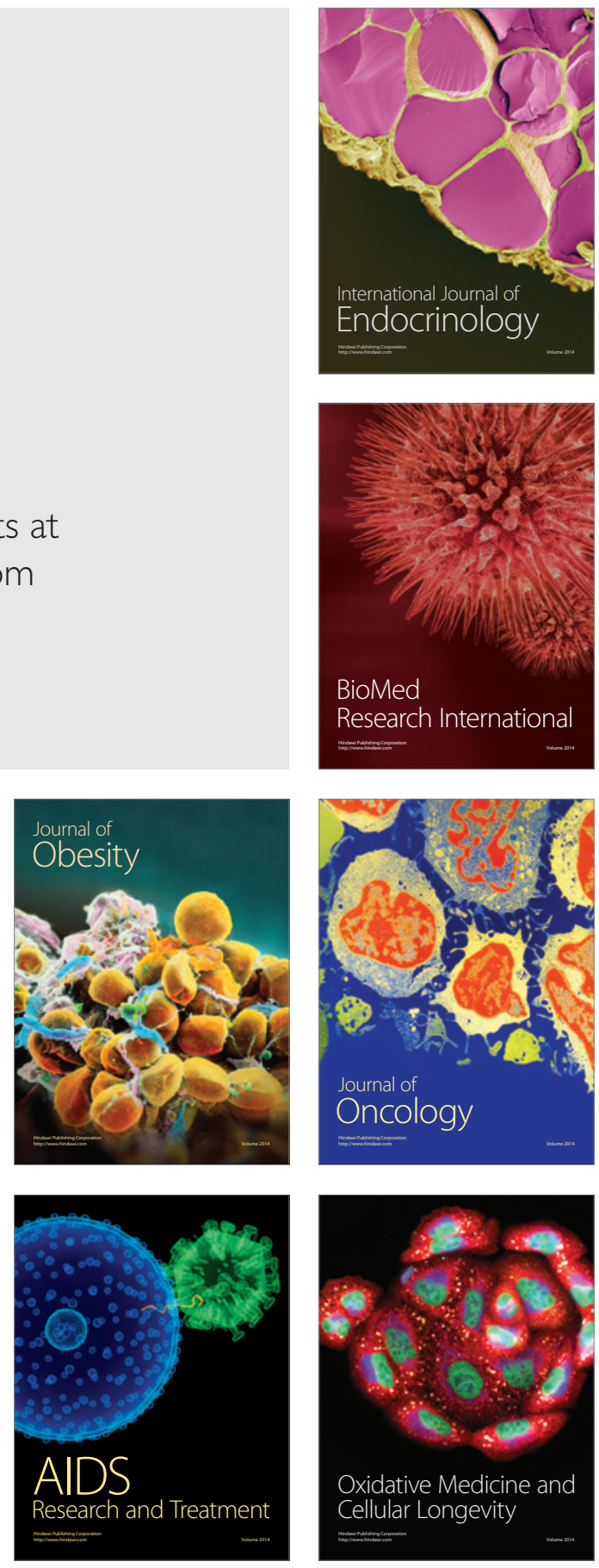